Conclusion: This study confirms that PM can now be considered a rare IIM subtype. A thorough examination, complete myositis antibody panel including HMGCR testing and careful interpretation of the biopsy results is recommended to accurately classify these patients.

References:

[1] Parker MJS, Oldroyd A, Roberts ME, Lilleker JB, Betteridge ZE, McHugh NJ, et al. The performance of the European League Against Rheumatism/American College of Rheumatology idiopathic inflammatory myopathies classification criteria in an expert-defined 10 year incident cohort. Rheumatol (United Kingdom). 2019 Mar 1;58(3):468-75

Disclosure of Interests: None declared

DOI: 10.1136/annrheumdis-2020-eular.1435

\section{SAT0332 ANTIBODIES AGAINST CYTOSOLIC 5'-NUCLEOTIDASE 1 A IN SPORADIC INCLUSION BODY MYOSITIS: ASSOCIATION WITH CLINICAL AND MRI FEATURES}

R. Dejthevaporn ${ }^{1}$, S. Shah ${ }^{2}$, S. Wastling ${ }^{2}$, J. Thornton ${ }^{2}$, T. Yousry ${ }^{2}$, J. M. Morrow ${ }^{1}$, P. M. Machado ${ }^{3} .{ }^{1}$ University College London, Queen Square Centre for Neuromuscular Diseases, London, United Kingdom; ${ }^{2}$ University College London, Neuroradiological Academic Unit, London, United Kingdom; ${ }^{3}$ University College London, Centre for Rheumatology \& Queen Square Centre for Neuromuscular Diseases, London, United Kingdom

Background: Autoantibodies directed against cytosolic 5'-nucleotidase $1 \mathrm{~A}$ (cN1A) have been identified in sporadic inclusion body myositis (SIBM) and other connective tissue diseases. Anti-cN1A antibodies may support the diagnostic process for sIBM as well as potentially provide clues for disease pathogenesis. Nevertheless, the utility of anti-cN1A autoantibody testing in clinical practice remains unclear and requires validation.

Objectives: To investigate the association between anti-cN1A antibody status and clinical and MRI features in patients with sIBM.

Methods: Data for patients fulfilling European Neuromuscular Centre (ENMC) 2011 criteria for sIBM were obtained from a natural history study database. Demographic, clinical, functional assessment, and muscle MRI data in patients with sIBM who had anti-cN1A autoantibody testing were collected and analysed. Comparisons between subgroups with anti-cN1A antibody status were performed with the Mann-Whitney or Fisher's exact tests, as appropriate.

Results: Forty-nine patients with sIBM had anti-cN1A autoantibody testing, of whom 17 (34.7\%) were positive. Twelve patients had muscle MRI performed (seropositivity=5). Demographics, disease duration at antibody testing and overall disease pattern were closely matched in antibody positive and negative cohorts. Dysphagia was more common in the seropositive subgroup (77\% vs $47 \%, p=0.070$ ). Antibody positive patients were more severely affected with a trend to lower IBM functional rating scale (IBMFRS) scores $(22.4 \pm 8.4$ vs $26.7 \pm 6.4, p=0.09)$ with significantly worse ability to climb stairs $(0.9 \pm 0.9,1.7 \pm 1.1$ $\mathrm{p}=0.02)$. On T1-weighted MRI more fatty infiltration was found in seropositive patients (Mercuri score: $3.0 \pm 0.8$ vs $1.7 \pm 0.7, p=0.03$ ). Short tau inversion recovery (STIR) hyperintensity was more conspicuous in seropositive patients (STIR extent score: $2.4 \pm 0.6$ vs $1.4 \pm 0.7, p=0.04$ )

Conclusion: There was a trend for more dysphagia and severity of dysphagia in seropositive patients. Differences in upper limb involvement were not seen according to IBMFRS and Medical Research Council (MRC) strength grades Seropositive patients were more severely affected at the lower limb level, in terms of muscle weakness, physical function, MRI fatty infiltration and muscle inflammation. These results suggest positive antibody status is associated with a worse phenotype. These results have potential implications in clinical trials: whether antibody status influences treatment response should be assessed.

Disclosure of Interests: Revadee Dejthevaporn: None declared, Sachit Shah: None declared, Stephen Wastling: None declared, John Thornton: None declared, Tarek Yousry: None declared, Jasper M Morrow: None declared, Pedro M Machado Consultant of: PMM: Abbvie, Celgene, Janssen, Lilly, MSD, Novartis, Pfizer, Roche and UCB, Speakers bureau: PMM: Abbvie, BMS, Lilly, MSD, Novartis, Pfizer, Roche and UCB

DOI: 10.1136/annrheumdis-2020-eular.3399

\section{SAT0333 SERUM METABOLITES AS BIOMARKERS IN SYSTEMIC SCLEROSIS-ASSOCIATED INTERSTITIAL LUNG DISEASE}

C. Meier ${ }^{1}$, K. Freiburghaus ${ }^{2}$, C. Bovet ${ }^{2}$, J. Schniering ${ }^{1}$, O. Distler ${ }^{1}$, C. Nakas ${ }^{2,3}$, B. Maurer ${ }^{1}{ }^{1}$ Center of Experimental Rheumatology, Department of Rheumatology, University Hospital Zurich, Zurich, Switzerland; ${ }^{2}$ University Institute of Clinical Chemistry, Inselspital, Bern University Hospital, University of Bern, Bern, Switzerland; ${ }^{3}$ Laboratory of Biometry, Department of Agriculture,
Crop Production and Rural Environment, School of Agricultural Sciences, University of Thessaly, Thessaly, Greece

Background: In fibrotic diseases, metabolic processes are altered with a tendency towards an anabolic state, which is partially reflected in serum. Circulating biomarkers for interstitial lung disease (ILD), the leading cause of death in systemic sclerosis (SSc), are still sparse and not established in routine care.

Objectives: To assess the potential of serum metabolites as biomarkers for the presence and progression of SSc-ILD.

Methods: Age and sex matched serum samples of SSc patients from the Zurich cohort and of healthy controls (HC) were analyzed. Progressive SSc-ILD was defined as either a relative decrease in forced vital capacity (FVC) $>10 \%$, a decrease in FVC of $5-9 \%$ and a concomitant decrease of carbon dioxide diffusion capacity $>15 \%$, or an increase of the extent of lung fibrosis on computed tomography from $<20 \%$ to $\geq 20 \%$ compared to the last visit (mean follow-up interval $=14$ months (range $=9-26)$ ). Sera of HC, non-ILD SSc and stable vs progressive SSc-ILD patients ( $n=12$ per group; total $n=48$ ) were screened for 110 metabolites by targeted liquid chromatography coupled to tandem mass spectrometry (LC-MS/MS). Peak areas were analyzed with R 3.6. For univariate analysis, FDR-corrected one-way ANOVA was used. In multivariate group-wise partial least squares discriminant analysis (PLS-DA), variable importance in the projection (VIP) scores $\geq 2$ were considered significant.

Results: In total, 85 metabolites were detected. Univariate analysis of all groups were suggestive of changes for 1-methyladenosine, L-tryptophan, L-tyrosine, L-leucine and xanthosine ( $p=0.077,0.028,0.077,0.028$ and 0.032 , respectively). In PLS-DA, HCs and SSc patients differed in their levels of L-tyrosine and L-tryptophan, while levels of L-threonine, 3-aminoisobutyric acid, adenosine monophosphate and xanthosine were changed when comparing non-ILD and SSc-ILD patients. Receive operating curve (ROC) analysis of significant metabolites from uni- and multivariate testing resulted in separation of SSc patients from $\mathrm{HCs}$ by L-tyrosine (area under the curve $(A \cup C)=0.81,95 \%$ confidence interval $(\mathrm{Cl})$ : 0.67-0.96), L-tryptophan (AUC = $0.86, \mathrm{Cl}: 0.75-0.97)$ and 1-methyladenosine (AUC $=0.82, \mathrm{Cl}: 0.71-0.94)$. Progressive SSc-ILD patients were separated from stable patients by their levels of L-isoleucine L-leucine, adenosine monophosphate and xanthosine (AUC $=0.83,0.85,0.79$ and 0.77 ; Cl: $0.66-1.00,0.70-1.00,0.60-0.97$ and 0.55-0.99, respectively). Validation of increased values of the branched-chain amino acids L-leucine and L-isoleucine in progressive SSc-ILD vs. stable ILD using an enzymatic assay resulted in simila results as LC-MS/MS analysis, with higher values detected in progressive vs. stable patients (mean $=286.5$ and $235.5 \mathrm{nM}$, respectively; $\mathrm{p}=0.005$ ). In ROC analysis (AUC $=0.81, \mathrm{Cl}: 0.62-1.00$ ), a cut-off value of $250.3 \mathrm{nM}$ separated stable from progressive patients with a sensitivity of $72.7 \%$ and a specificity of $83.3 \%$.

Conclusion: This study in SSc(-ILD) patients suggested alterations in serum metabolite levels corresponding with their current state of disease, indicating the potential use of serum metabolites as discriminating biomarkers upon furthe confirmation in larger multicenter studies.

Disclosure of Interests: Chantal Meier: None declared, Katrin Freiburghaus: None declared, Cédric Bovet: None declared, Janine Schniering: None declared, Oliver Distler Grant/research support from: Grants/Research support from Actelion, Bayer Boehringer Ingelheim, Competitive Drug Development International Ltd. and Mitsubishi Tanabe; he also holds the issued Patent on mir-29 for the treatment of systemic sclerosis (US8247389, EP2331143)., Consultant of: Consultancy fees from Actelion, Acceleron Pharma, AnaMar, Bayer, Baecon Discovery, Blade Therapeutics, Boehringer, CSL Behring, Catenion, ChemomAb, Curzion Pharmaceuticals, Ergonex, Galapagos NV, GSK, Glenmark Pharmaceuticals, Inventiva, Italfarmaco, iQvia, medac, Medscape, Mitsubishi Tanabe Pharma, MSD, Roche, Sanofi and UCB, Speakers bureau: Speaker fees from Actelion, Bayer, Boehringer Ingelheim, Medscape, Pfizer and Roche, Christos Nakas: None declared, Britta Maurer Grant research support from: AbbVie, Protagen, Novartis, congress support from Pfizer Roche, Actelion, and MSD, Speakers bureau: Novartis DOI: 10.1136/annrheumdis-2020-eular.702

\section{SAT0334 PERICARDIAL INVOLVEMENT IN SYSTEMIC SCLEROSIS}

P. Morán Álvarez ${ }^{1}$, C. De la Puente Bujidos ${ }^{1} .{ }^{1}$ Hospital Universitario Ramón y Cajal, Madrid, Spain

Background: Systemic sclerosis (SSc) is an autoimmune rheumatic disease characterized by visceral and skin fibrosis, vascular dysfunction, and immune dysregulation. Regarding cardiac manifestations, pericardial disorder is one of the most frequent but often asymptomatic.

Objectives: To analyze clinical manifestations, diagnostic tools and treatments of a patient cohort with SSc and pericardial involvement associated.

Methods: A descriptive, observational, cross-sectional study was carried out We included all patients between 1975 and 2019 with diagnosis of SSc. Demographic, clinical and analytical data; imaging tests; treatments; and mortality rate were collected. 